\title{
Interferometric Observations of Disks around PMS Binary Stars
}

\author{
Anne Dutrey \\ Institut de Radio Astronomie Millimétrique 300 rue de la picine, \\ F-38406, Saint-Martin-d'Hères, France
}

\begin{abstract}
Current millimetre arrays are well suited to study the gas and dust distribution around young binary systems of separation $\sim 0.3-$ $3^{\prime \prime}$. In particular, they clearly demonstrate that disks around binary systems are tidally truncated. I review here the observed properties at $\mathrm{mm}$ wavelengths of the gas and dust around the best known multiple systems. I also discuss the limitations of current $\mathrm{mm}$ interferometers.
\end{abstract}

\section{Introduction}

In this review, I focus on the observed properties at $\mathrm{mm}$ wavelengths of the gas and dust around Pre-Main-Sequence (PMS) low mass binary stars (the socalled Class II phase). These stars are mainly visible objects of a few Myr old. Contrary to the proto-binary stage (see the review by L.Mundy), most of their surrounding envelope of gas and dust is already dissipated and their outflow activity remains weak.

Recent $\mathrm{mm}$ and submm surveys (Osterloh \& Beckwith 1995, Jensen et al. 1996a, Dutrey et al. 1996) show that the mm flux densities of binary PMS stars are usually significantly weaker than those of single stars (see also the review by E.Jensen, this conference). Even if recent models of collapsing molecular cloud cores (Bates et al. 2000) suggest that the properties of the circumbinary (CB) material (e.g. the existence of a CB disk) would be dependent on the initial conditions within the parent cores (see the review by M.Bates, this conference), it is however widely accepted that observed differences in flux density can be simply explained by tidal truncation rather than by different surface density laws in disks around singles and binaries (Artymowicz et al. 1991).

In a simplistic scheme, the circumstellar (CS) material around a binary should be separated in four main regions: two possible circumstellar disks within the Roche lobes and surrounding the individual stars (expected to be truncated at $0.2-0.5$ times the binary separation), an "emptied" cavity or gap and a circumbinary disk lying outside the $\mathrm{L}_{2}$ and $\mathrm{L}_{3}$ Lagrangian points (of inner radius 2-3 times the separation). Moreover since CS disks are small, and assuming standard surface density laws, they would be rapidly exhausted by accretion on stars. Therefore there must be, at least for close separations, a mechanism which replenishes the CS disks from a CB disk or an envelope (Artymowicz \& Lubow 1994) to allow them to survive a Myr or more. 

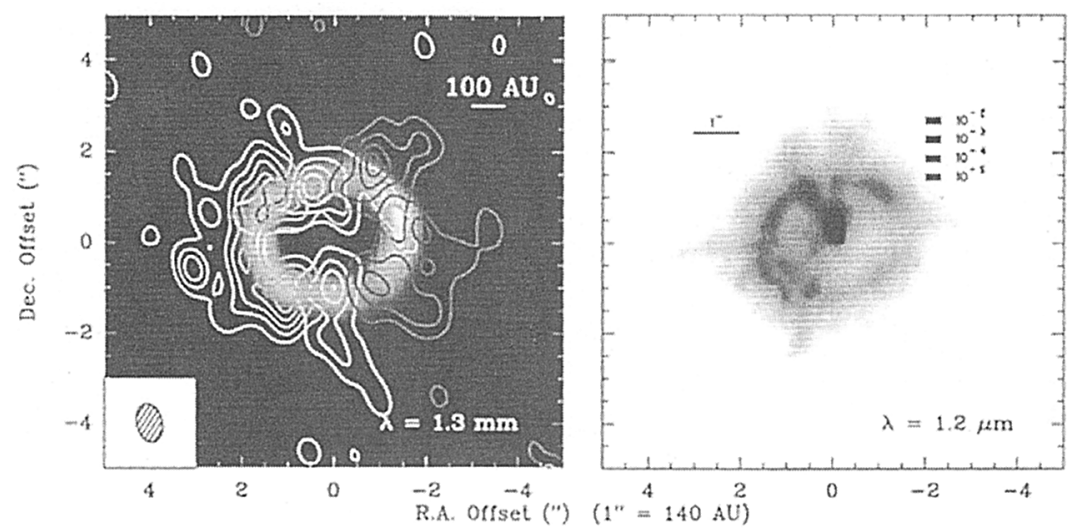

Figure 1. Left: IRAM data, ${ }^{13} \mathrm{CO} \mathrm{J}=2-1$ (Guilloteau et al. 1999) line emissions at 5.55, 6.30 (systemic velocity) and $7.05 \mathrm{~km} . \mathrm{s}^{-1}$, overlaid on a false colour image of the continuum emissions at $1.4 \mathrm{~mm}$. For the $1.3 \mathrm{~mm}$ data, the beam size is $0.6^{\prime \prime} \times 0.9^{\prime \prime}$ at P.A. $13^{\circ}$. Right: NIR observations from Roddier et al. 1996 in the $\mathrm{J}$ band. The forward scattering dominates the emission and the northern part of the disk points towards us, in agreement with the binary orbit and the Keplerian rotation.

\section{Observed Morphology of Material around Multiple T Tauri Systems}

With typical temperature of $\sim 100-200 \mathrm{~K}$ at $10-30 \mathrm{AU}$ from the star, material around low-mass TTauri stars is relatively cold. Except at a few AU from the central engine, it mainly radiates at $\mathrm{mm}$ and submm wavelengths. Therefore high angular resolution $\left(0.5-3^{\prime \prime}\right)$ images from current $\mathrm{mm}$ arrays are well suited to study the dust and gas distribution around binaries of separation around $0.3-3^{\prime \prime}$. The three following examples are among the best known multiple systems showing both circumstellar and/or circumbinary material. They correspond to different angular separations and illustrate the wide range of observed morphologies.

\subsection{GG Tau: A Case Study ?}

GG Tau is the first example of resolved circumbinary disk in very good agreement with tidal truncation expectations. This classical TTauri star is a binary of separation $0.26^{\prime \prime}$ in the plane of the sky. At $1.3 \mathrm{~mm}$, its continuum flux is $600 \mathrm{mJy}$, well above what is usually found around such stars. There is indeed a 
very massive $\left(0.15 \mathrm{M}_{\odot}\right)$ and large $\mathrm{CO}$ and dust $\mathrm{CB}$ disk which was first resolved by $\mathrm{mm}$ interferometry (Dutrey et al. 1994, Guilloteau et al. 1999). This disk, also imaged in scattered light in the near-infrared (NIR) by Roddier et al. (1996), appears to be dominated by a ring in Keplerian rotation and has an inner hole of radius $R_{i n} \simeq 180 \mathrm{AU}$. The size of the central hole, the P.A. and the inclination angle of the disk determined both from the $\mathrm{mm}$ and NIR data are in very good agreement, demonstrating that the dust observed 1) in the thermal $\mathrm{mm}$ regime and 2) in the scattering regime, is mixed, and that 3) the $\mathrm{CO}$ emission also originates from the same disk. Recently, Silber et al. (2000) have obtained HST polarimetric maps of the disk at $1 \mu \mathrm{m}$; these data are in good agreement with the previous ones and like the mm data support the hypothesis of a thick ring. Finally, using the IRAM interferometer, Guilloteau and collaborators have detected $\mathrm{CO} \mathrm{J}=2-1$ emission within the gap, suggesting that some gas and dust could flow from the CB disk into the CS disks (see also the proceeding by S.Guilloteau, this conference, for a complete description of the GG Tau system).

\subsection{UY Aur: A Common 0.9" Binary ?}

UY Aur is a Classical TTauri binary of separation $\sim 0.89^{\prime \prime}$. Using the IRAM array, Duvert et al. (1998) have observed in ${ }^{13} \mathrm{CO} \mathrm{J}=1-0$ and $\mathrm{J}=2-1$ lines a $\mathrm{CB}$ ring in Keplerian rotation around the stars while the continuum emission at $1.3 \mathrm{~mm}$ peaks on the binary itself and is associated with the CS disks. Further NIR observations performed by Close et al. (1998, their fig.1) confirm the existence of the CB ring. NIR polarimetric maps obtained at the CFHT by Potter et al. (2000) also confirm the ring model. The ring mass, roughly estimated from the ${ }^{13} \mathrm{CO}$ data is about $\sim 3-7 \cdot 10^{-3} \mathrm{M}_{\odot}$.

With a less massive CB ring $\left(M \leq 10^{-2} \mathrm{M}_{\odot}\right)$ which is not detected in continuum at $1.3 \mathrm{~mm}$, the gas and dust distribution around UY Aur is more likely representative of disks around binaries than the GG Tau circumbinary disk which remains exceptionally massive $\left(0.15 \mathrm{M}_{\odot}\right)$.

\subsection{UZ Tau: A Quadruple System}

UZ Tau includes a wide East-West binary of separation 3.4". Both components are themselves binary stars of separation $0.34^{\prime \prime}$ for UZ Tau West, UZ Tau East being a spectroscopic binary with $a \cdot \sin (i) \simeq 0.03 \mathrm{AU}$ (Mathieu et al. 1996). Jensen et al. 1996b observed UZ Tau with the OVRO interferometer in ${ }^{12} \mathrm{CO} 2-$ 1 and in continuum at $1.3 \mathrm{~mm}$. These observations (see their fig. 1 and fig.2) show that strong continuum and $\mathrm{CO}$ emissions arise from the spectroscopic binary UZ Tau East. The CO pattern suggests rotation. On UZ Tau West, the continuum emission is weaker and unresolved. Fig.2 (Dutrey et al. 2001, in prep.) shows the ${ }^{12} \mathrm{CO} \mathrm{J}=2-1$ emission map from UZ Tau obtained with the IRAM array. This clearly confirms Keplerian rotation, and also reveals the existence of a weak $\mathrm{CO}$ emission on UZ Tau West, more likely associated to possible CS disks.

The UZ Tau case illustrates the complexity of multiple systems and the fact that contrarily to a close separation, a wide separation (here $\sim 3.4^{\prime \prime}$ or $\sim 500$ $\mathrm{AU}$ ) allows to find CS disks (even in CO) around individual components (UZ Tau East). Moreover, UZ Tau East was recently identified as a spectroscopic 


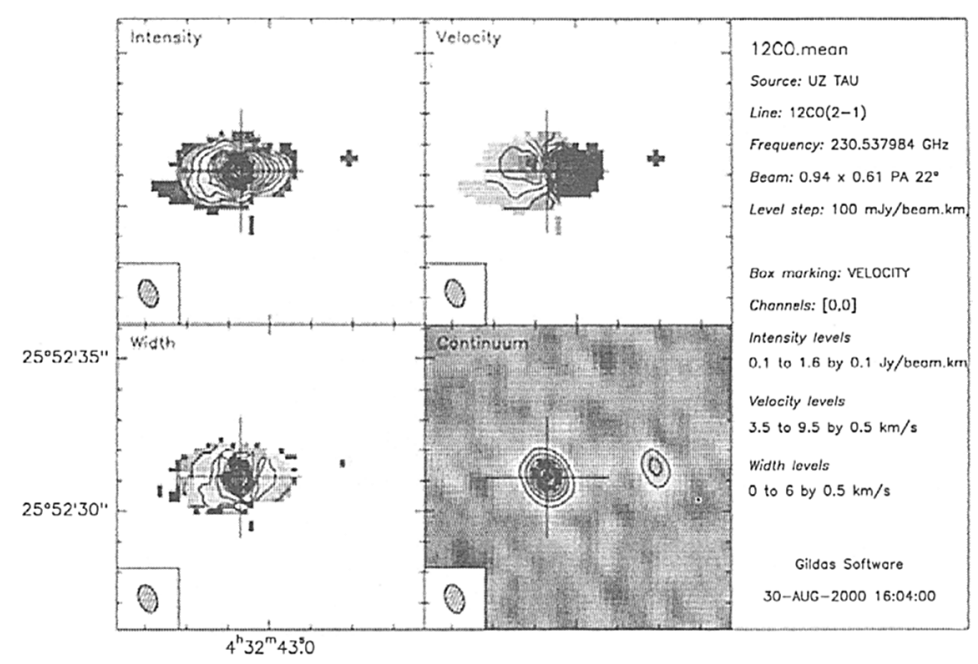

Figure 2. UZ Tau: a) integrated area, b) velocity gradients, c) linewidth of the ${ }^{12} \mathrm{CO} \mathrm{J}=2-1$ line and d) continuum emission at $1.3 \mathrm{~mm}$, interferometric observations from the IRAM array (Dutrey et al. 2001, in prep.). Dust emission is clearly detected on both binaries. On CO maps, the small blob on the west, above the noise level, should correspond to $\mathrm{CO}$ emission associated to UZ Tau West, the binary of separation $0.34^{\prime \prime}$. Unresolved, this emission is more likely associated to possible inner disks.

binary. This does not change its expected $\mathrm{mm}$ flux (separation $<1 \mathrm{AU}$ ) but it reminds us that many other "robust" single stars could be spectroscopic binaries.

\section{Imaging Disks around Binaries with mm Arrays}

Fig.3 explains qualitatively how the material is distributed around a binary system and what regions can easily be detected with an interferometer. More explanations about how this figure is obtained and should be understood can be found in Dutrey 2000.

For a binary of separation $0.5-0.6^{\prime \prime}$, the gap created by tidal effects would be located between $\sim 0.3^{\prime \prime}$ and $\sim 2^{\prime \prime}$. Hence the interferometer would easily detect the unresolved continuum emission coming from the circumstellar inner dust disks (if any) and the $\mathrm{CO}$ emission associated to the CB disk. This is exactly what happens in the case of the UY Auriga system. The CO emission associated to the inner disks would be extremely hard to detect due to the beam dilution effect, even if it seems plausible for UZ Tau West. Finally, the dust in the CB disk escapes detection because its emission is too optically thin and its brightness temperature is below the detection threshold of the array. This simulation assumes that the disk $(\mathrm{CB}+\mathrm{CS})$ is relatively massive $\left(0.05 \mathrm{M}_{\odot}\right)$ and the CB disk is in this case marginally detected in continuum. For a massive disk $\left(\sim 0.15 \mathrm{M}_{\odot}\right)$ such as the GG Tau ring, continuum detection is clearly possible 
because the dust opacity is high enough (but still optically thin at $1.3 \mathrm{~mm}$ ) to allow mapping of the brightness distribution.

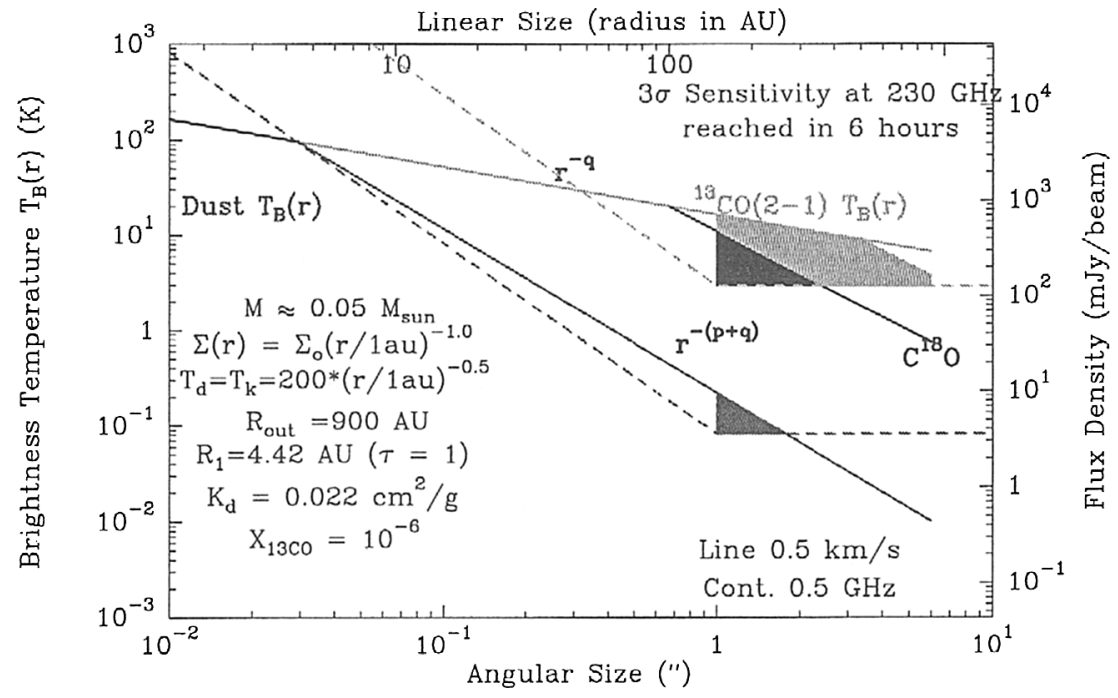

Figure 3. Brightness distribution in Kelvin (Rayleigh-Jeans approximation) of dust at $1.3 \mathrm{~mm}$ (dark grey), ${ }^{13} \mathrm{CO} \mathrm{J}=2-1$ (grey) and $\mathrm{C}^{18} \mathrm{O}$ $\mathrm{J}=2-1$ (black) lines in a disk and comparison with the sensitivity curves of the IRAM interferometer at $3 \sigma$ for the continuum (dashed line in dark grey) and the lines (dashed line in light grey). The resolution of the array is $1.0^{\prime \prime}$. The scale is $\log$-log and the angular size $(")$ is scaled to the Taurus distance $(150 \mathrm{pc})$ with radius in AU. The interferometer is only sensitive to disk emission above the sensitivity curves. Filled areas correspond to the regions of the disk where the emissions are detectable and resolved. Differences between $\mathrm{C}^{18} \mathrm{O}$ and ${ }^{13} \mathrm{CO}$ emissions come from the fact that $\mathrm{C}^{18} \mathrm{O}$ is less abundant than ${ }^{13} \mathrm{CO}$. Tidal truncation for a system having a separation around $0.5-0.6^{\prime \prime}$ would create a gap inside the distribution around $\sim 0.3-2^{\prime \prime}$. Only the inner dust disks would be detected in continuum.

Table 1 summarizes typical flux densities for circumstellar and circumbinary disks around binary stars. The flux density of an optically thick inner disk of outer radius $20 \mathrm{AU}$ and temperature $45 \mathrm{~K}$ is $70 \mathrm{mJy}$ at $1.3 \mathrm{~mm}$, which is easily detected by most $\mathrm{mm}$ arrays. In the same conditions, with an integrated flux around $25 \mathrm{mJy} \cdot \mathrm{km} / \mathrm{s}$, the $\mathrm{CO} \mathrm{J}=2-1$ line would be undetectable. Hence CS inner disks can be detected in continuum by $\mathrm{mm}$ arrays but fail to be detected in CO lines because they are beam diluted.

On the contrary, circumbinary disks observed with current arrays should be only detectable in nearly optically thick CO lines. A $0.01 \mathrm{M}_{\odot} \mathrm{CB}$ dust disk (assuming a gas/dust ratio of 100) of inner and outer radius 200 and $400 \mathrm{AU}$, respectively and temperature of $15 \mathrm{~K}$ would radiate $40 \mathrm{mJy}$ in optically thin continuum at $1.3 \mathrm{~mm}$. Since such a disk is extended on about 10 beams for a $1^{\prime \prime}$ resolution, the detected flux would be $4 \mathrm{mJy} /$ beam corresponding to a $4 \sigma$ 
detection (upper limit) for standard sensitivity of the IRAM array. The same gas disk would radiate $8 \mathrm{Jy} . \mathrm{km} / \mathrm{s}$ in the optically thick $\mathrm{CO} \mathrm{J}=2-1$ line, well above the detection threshold of the interferometer. This is fully consistent with the detection of the UY Aur CB disk in ${ }^{13} \mathrm{CO}$, only.

Table 1.

\begin{tabular}{|c|c|c|}
\hline $\begin{array}{c}\text { Disk/Ring } \\
1.3 \mathrm{~mm}\end{array}$ & $\begin{array}{c}\text { Dust emission } \\
(\mathrm{Jy})\end{array}$ & $\begin{array}{c}\mathrm{CO} J=2-1 \text { line } \\
(\mathrm{Jy} \cdot \mathrm{km} / \mathrm{s})\end{array}$ \\
\hline $\begin{array}{c}\text { inner disk } \\
r_{\text {out }}=20 \mathrm{AU} \\
T_{k}=45 \mathrm{~K}\end{array}$ & $\begin{array}{l}\sim 0.07 \\
\text { opt. } \\
\text { thick }\end{array}$ & $\begin{array}{l}\sim 0.025 \\
\text { opt. } \\
\text { thick }\end{array}$ \\
\hline $\begin{array}{c}\text { ring } \\
r_{\text {in }}=200 \mathrm{AU} \\
r_{\text {out }}=800 \mathrm{AU} \\
T_{k}=15 \mathrm{~K}\end{array}$ & $\begin{array}{c}\sim 0.04 \\
\sim 4 \text { mJy } / \text { Beam } \\
\quad\left(\text { or } 4 \sigma, 1^{\prime \prime}\right) \\
M_{\text {ring }}=0.01 \mathrm{M}_{\odot}\end{array}$ & $\begin{array}{l}\sim 8.0 \\
\text { opt. } \\
\text { thick }\end{array}$ \\
\hline $\begin{array}{c}\text { For comparison } \\
\text { DM Tau disk } \\
r_{\text {out }} \simeq 850 \mathrm{AU} \\
M_{\text {disk }} \sim 0.01 \mathrm{M}_{\odot}\end{array}$ & 0.11 & 12.5 \\
\hline
\end{tabular}

Note to table 1

Local line-width: $\delta v=0.3 \mathrm{~km} / \mathrm{s}$, line-width at half power: $D V_{K e p l e r}=2 \mathrm{~km} / \mathrm{s}$

\section{Observed Properties at $\mathrm{mm}$ Wavelengths}

There are now many examples of objects mapped at $1.3 \mathrm{~mm}$ and/or in CO lines with $\mathrm{mm}$ arrays. I will review here the observed properties of objects which are expected to be representative of the binary stars.

\subsection{Properties of Inner Circumstellar Disks}

Until recently, most of the detection of inner dust disks were obtained in continuum because $\mathrm{CO}$ line observations with current $\mathrm{mm}$ arrays are limited by sensitivity.

At mm wavelengths, the origin of the unresolved circumstellar continuum is mostly thermal and dominated by the emission of the dust. Assuming a standard surface density distribution $\Sigma(r) \propto r^{-p}$ with $p=1--1.5$, the dust emission of the circumstellar inner disks should be optically thick at $1.3 \mathrm{~mm}$ within a radius of $10-20 \mathrm{AU}$.

One cannot ignore a possible weak contribution of free-free emission coming from a jet, particularly at $\lambda=7 \mathrm{~mm}$ (e.g. the disk around the single star DO Tau, Koerner et al. 1995). In the case of V773 Tau, a binary star of separation $0.1^{\prime \prime}$, Dutrey et al. (1996) have reported variable emission at $3 \mathrm{~mm}$ on the scale of a few months. Since this binary has a strong magnetic activity (Feigelson et al. 1994), the origin of such a variability might arise from magnetospheric interactions (synchrotron emission). 
Tidal Truncation Fig.4 shows $1.3 \mathrm{~mm}$ dust maps of several TTauri disks obtained with the IRAM array. Tidal truncation of inner disks is clearly observed in several cases for binary having separation around $0.3-1^{\prime \prime}$.

The images show that all disks are resolved, except the two CS disks of Haro 6-10, a binary star of separation $1.3^{\prime \prime}$. The size of both disks is significantly smaller than those of disks fround around singles (e.g. DM Tau, GM Aur, LkCa15...) and in agreement with tidal truncation of the outer radii.

One should also mention the disk around TTauri North (separation of $0.7^{\prime \prime}$ ). TTauri South has been recently identified as a binary of separation 10-15 AU (Koresko et al. 2000). Such a separation should easily explain the weak $1.3 \mathrm{~mm}$ flux which is observed, the gap being located at the distance from the binary where most of the $1.3 \mathrm{~mm}$ emission would otherwise come. This situation is similar to that of UZ Tau where most of the mm flux is located on the spectroscopic binary, not on the close binary (UZ Tau West of separation $0.34^{\prime \prime}$ ).

Finally, the CO and dust disk of UZ Tau East has a size compatible with tidal truncation by the wide binary UZ Tau of separation $3.4^{\prime \prime}$.

Coplanarity The poor angular resolution of current mm arrays does not allow yet to directly measure the inclination angles of inner disks. One should wait for the high angular resolution of ALMA. We however have an indirect measurement in the case of the spectroscopic binary UZ Tau East where the orbit of the spectroscopic binary appears not coplanar with its circumbinary disk (Simon et al. 2000). Another indirect evidence of non coplanarity might come from the existence of quadrupolar jets detected at $\mathrm{cm}$ wavelengths with the VLA (Rodriguez 1997).

Physical Parameters For close separation $\left(0.1-1^{\prime \prime}\right)$ inner dust disks are expected to be mostly optically thick, even at $1.3 \mathrm{~mm}$. Detailed studies require not only high angular resolution images in the $\mathrm{mm}$ and submm range which are beyond the scope of what $\mathrm{mm}$ arrays can achieve today but also multi-wavelength analysis.

One should however cite the detailed study performed by Akeson and collaborators (1998) on TTauri North. Using images at 7, 3 and $1.3 \mathrm{~mm}$ and assuming a standard disk model in power laws, they determine a dust spectral index $\beta=0.54_{-0.17}^{+0.27}$ and an outer disk radius $r_{\text {out }}=41_{-14}^{+26} \mathrm{AU}$ for a total disk mass of $\log \left(M / \mathrm{M}_{\odot}\right)=-2.4_{-0.6}^{+0.7}$ or $\sim 4 \cdot 10^{-3} \mathrm{M}_{\odot}$. These results are not inconsistent with the properties of the inner parts of disks found around single stars, however they are strongly limited by the sensitivity and the angular resolution.

\subsection{Properties of the Circumbinary Material}

As explained in $\S 3$, current interferometric surveys of binary stars fail to reveal circumbinary disks because they are strongly sensitivity limited. Circumbinary disks are imaged only around GG Tau, UY Aur and the spectroscopic binary UZ Tau East.

Only the GG Tau ring data present high enough sensitivity and resolution to allow detailed studies (S.Guilloteau, this conference). This ring remains however unique because it is among the most massive disk $\left(\sim 0.15 \mathrm{M}_{\odot}\right)$ found around 

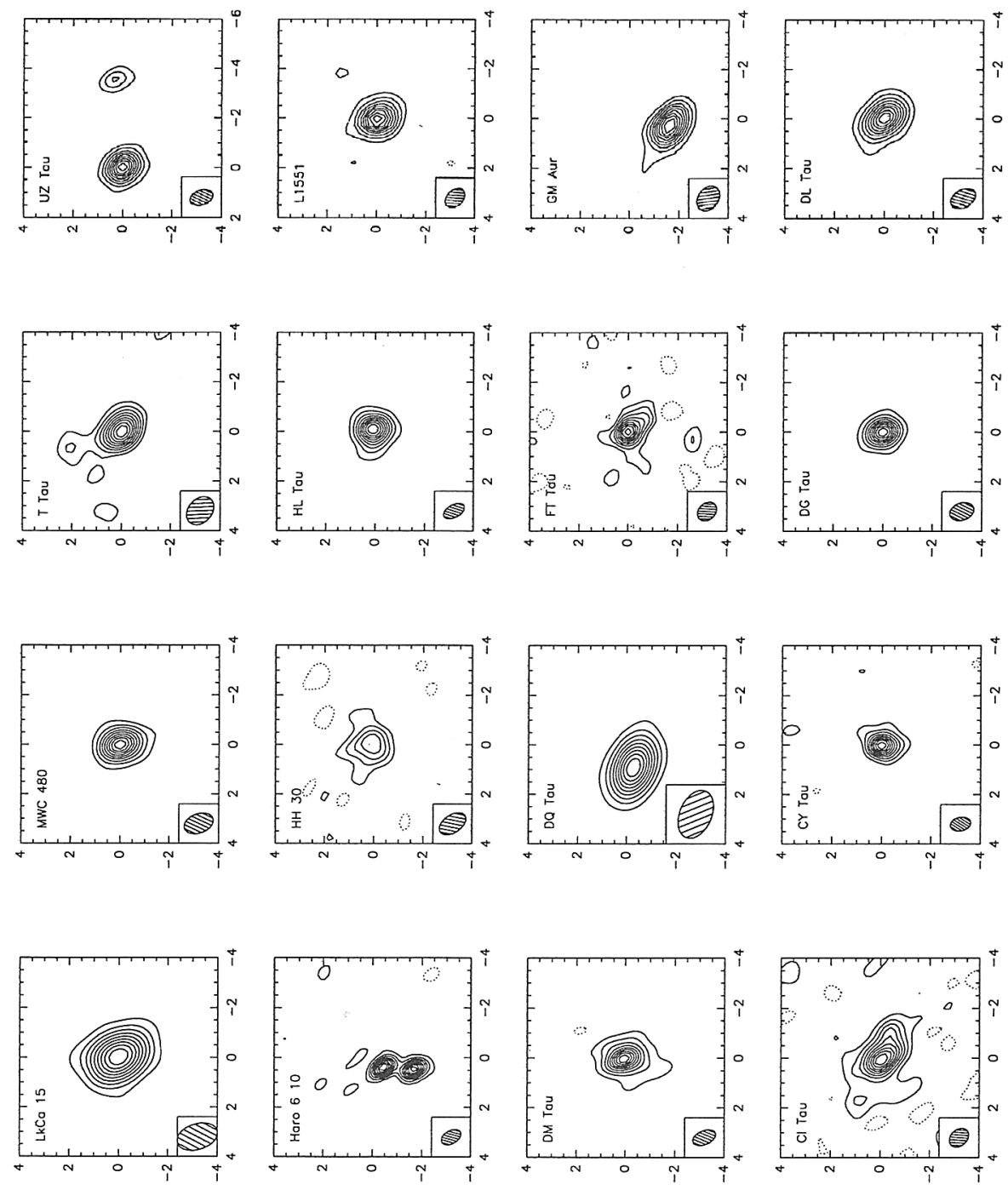

Figure 4. Survey at $1.3 \mathrm{~mm}$ of Tauri stars (single + multiple systems) obtained with the IRAM array (Dutrey et al. 2001, in prep.). The dust distribution around the binary star Haro 6-10 of separation $1.3^{\prime \prime}$ clearly shows that the inner disks are tidally truncated. For TTauri, the dust emission originates from the northern component (separation $0.7^{\prime \prime}$ in the plane of the sky). Contours are $10 \%$ of the peak, except for HH30 $(20 \%)$. 
a TTauri star. One should mention that most of the CB material $(70 \%)$ is confined in a narrow ring of width $80 \mathrm{AU}$ with very sharp inner edge $(\sim 10 \mathrm{AU})$. Confusion with the surrounding cloud does not allow Duvert et al. (1997) to perform a detail analysis of the properties of the circumbinary disk surrounding UY Auriga. However, with a mass around $\sim 0.01 \mathrm{M}_{\odot}$, this large disk (outer radius $\sim 800-1000 \mathrm{AU}$ ) is more likely representative of $\mathrm{CB}$ material than the GG Tau ring. It is not very different from the outer part of disks found around single stars (such as DM Tau, GM Aur or LkCa15).

The UZ Tau East CB disk has been analyzed by Dutrey et al. (2001). From the $\mathrm{CO} \mathrm{J}=2-1$ optically thick line, they deduce a temperature law of $\sim 30 \times(r / 100 A U)^{-0.64}$, in agreement from the stellar heating on a flared disk. Indeed, its physical properties appear very close to those found for outer part of disks around single stars.

\section{Summary}

Current $\mathrm{mm}$ arrays would allow to image circumbinary material surrounding binary systems of separation $0.3-1^{\prime \prime}$ if they were not strongly limited by sensitivity. There exist however a few example of imaged disks around binary and multiple systems. These maps clearly show that tidal interactions with the stars is the physical process responsible for the disk truncation, e.g GG Tau for the circumbinary disk or Haro 6-10 for the truncation of the inner disks. However, the non detection of circumbinary disks also clearly show that most of them have low masses $\sim 0.01 \mathrm{M}_{\odot}$, similar to those found around single stars. With a mass of $0.15 \mathrm{M}_{\odot}$, the GG Tau ring remains unique.

Detailed analysis of inner disks are beyond the scope of current $\mathrm{mm}$ arrays because they require very high angular resolution. One should wait for ALMA to get the high sensitivity and resolution maps needed to study the physical properties of outer and inner disks around binary systems.

Acknowledgments. I warmly thank S.Guilloteau and M.Simon for a fruitful long term collaboration. E.Jensen and F.Roddier are thanked for providing figures.

\section{References}

Akeson, R. L., et al. 1996, ApJ, 505, 358

Artymowicz, P. 1991 ApJ, 370, 35

Artymowicz, P., \& Lubow, S. 1994, ApJ, 421, 651

Bates, M. 2000, MNRAS, in press

Close, L., et al. 1997 ApJ, 499, 883

Duvert, G., et al. 1998, A\&A, 332, 867

Dutrey, A., Guilloteau, S., Simon, M. 1994, A\&A, 286, 149

Dutrey, A., et al. 1996, A\&A, 309, 493

Dutrey, A., Guilloteau, S., Guélin, M. 1997, A\&A, 317, L55 
Dutrey, A., in Science with the Atacama Large Millimetre Array, ASP Conf. Series

Dutrey, A., et al. 2001, in prep.

Feigelson et al. 1994, ApJ, 432, 373

Guilloteau, S., Dutrey, A. 1998, A\&A, 339, 467

Guilloteau, S., Dutrey, A., Simon, M. 1999, A\&A, 348, 57

Jensen, E., et al. 1996a, ApJ, 458, 312

Jensen, E., et al. 1996b, AJ, 111, 243

Koerner, D., et al. 1995, ApJ, 452, L69

Koresko, C., et al. 2000, ApJ, 531, L147

Mathieu, R., et al. 1996, Am.Astron.Soc., 188, 6005

Osterloh, M., \& Beckwith, S. W. 1995, A\&A, 439, 288

Potter, D., et al. 2000, ApJ, in press

Roddier, C., et al. 1996, ApJ, 463, 326

Rodriguez 1997, Herbig-Haro flows and the birth of low mass stars, IAU Symp. 197,83

Simon, M., Dutrey, A., Guilloteau, S. 2000, ApJ, in press

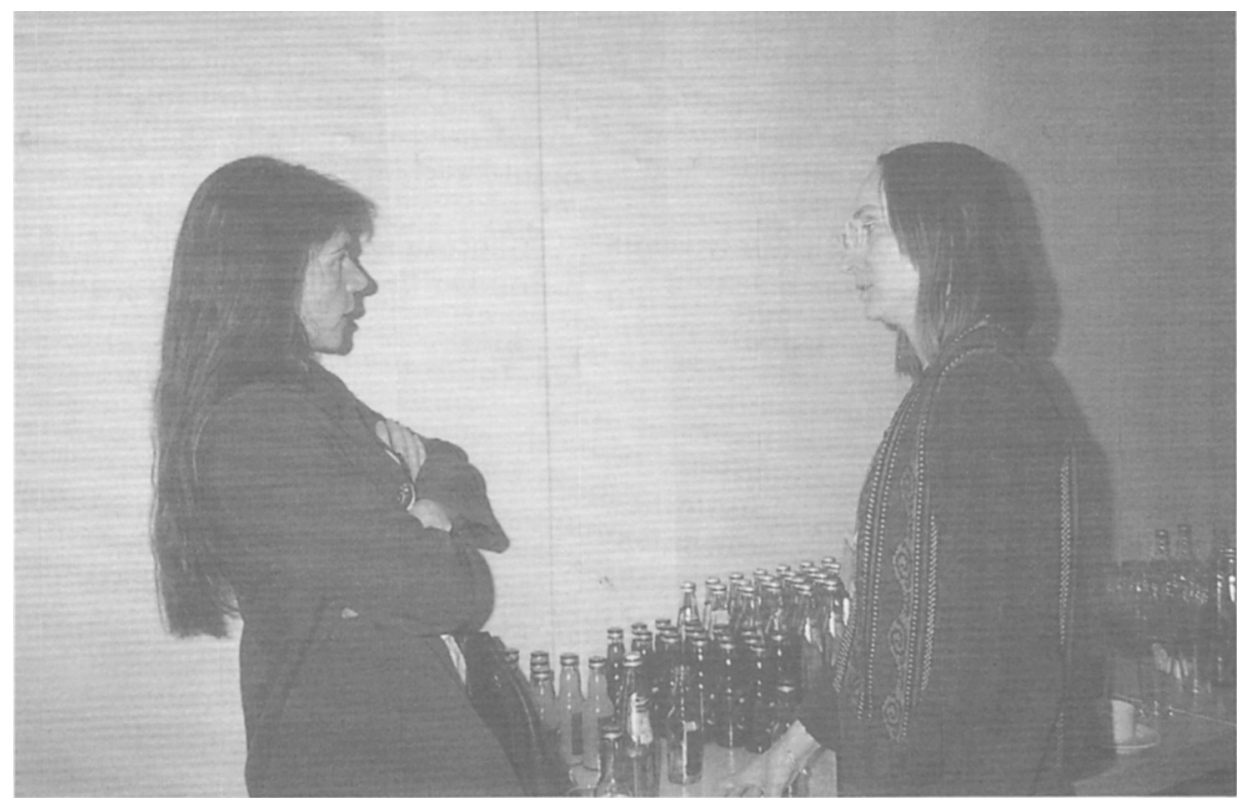

Isabelle Baraffe and Anne Dutrey 